Referencia para citar este artículo: López-Gallego, L., Galeotti-Galmés, R., \& Montes-Maldonado, C. (2018). Gestión de las sexualidades en los sistemas penales: las adolescentes mujeres. Revista Latinoamericana de Ciencias Sociales, Niñez y Juventud, 16(1), 413-426. doi:10.11600/1692715x.16126

\title{
Gestión de las sexualidades en los sistemas penales: las adolescentes mujeres*
}

\author{
LAURA LÓPEZ-GALLEGO ${ }^{\text {** }}$ \\ Profesora Universidad de la República, Uruguay. \\ RAQUEL GALEOTTI-GALMÉS ${ }^{* * *}$ \\ Profesora Universidad de la República, Uruguay. \\ CECILIA MonTES-MALDONADO \\ Profesora Universidad de la República, Uruguay.
}

\section{Artículo recibido en junio 22 de 2017; artículo aceptado en agosto 29 de 2017 (Eds.)}

- Resumen (descriptivo): en el artículo reflexionamos sobre la gestión de las sexualidades de las adolescentes mujeres tomando como punto de partida el Sistema Penal Juvenil Uruguayo. Trabajamos con una perspectiva de investigación cualitativa que apuesta a la producción de sentidos por parte de los y las agentes claves, a lo que sumamos los aportes de las epistemologías y las criminologías feministas. Presentamos dos tensiones analíticas, una referida a la sexualización del cuerpo y avasallamiento de la sexualidad. Y otra acerca del tratamiento de la maternidad de las adolescentes vinculado al pecado y el ejercicio de la sexualidad. Finalmente mostramos cómo muchas de las intervenciones se apuntalan en el cuerpo, al configurarse prácticas de control sociopenal destinadas a las sexualidades de las adolescentes mujeres.

Palabras clave: delincuencia juvenil, mujeres adolescentes, sexualidad (Tesauro de Ciencias Sociales de la Unesco).

Este artículo corto se basa en la línea investigación “Tratamiento socio-penal de las adolescentes mujeres en el Sistema Penal Juvenil Uruguayo, desde una perspectiva jurídica y de género", que llevan adelante las tres autoras Raquel Galeotti-Galmez, Laura López-Gallego y Cecilia MontesMaldonado. Se desarrolla en el marco del Grupo de Investigación y Desarrollo "Programa de Estudio sobre control socio-jurídico de infancia y adolescencia en Uruguay. Estudios sobre infracción adolescente", coordinado por las Dras. Carolina González y Sandra Leopold y el cual fue financiado por la Comisión Sectorial de Investigación Científica de la Universidad de la República, a partir del primero de marzo de 2015 y hasta el 28 de febrero de 2019 (Resolución No 8: Consejo Directivo Central, febrero de 2014). Área: Sociología; subárea: temas especiales.

** * * Licenciada en Psicología (2000) por la Facultad de Psicología de la Universidad de la República (UR). Magíster en Psicología Social (2006) por el Departamento de Psicología Social de la Universidad Autónoma de Barcelona (UAB). Doctora en Psicología (2016) por la Universidad Autónoma de Barcelona. Docente e investigadora del Instituto de Psicología Social de la Facultad de Psicología de la UR. Investigadora activa del Sistema Nacional de Investigadores de la Agencia Nacional de Investigación e Innovación (Anii). Trabaja líneas de investigación que vinculan género, sistemas penales juveniles y prácticas psi. Orcid: 0000-0003-3413-8537. Índice H5: 2. Correo electrónico: 1lopez@psico.edu.uy

*** Licenciada en Psicología (1997) por la Facultad de Psicología de la Universidad de la República (UR). Magíster en Derechos de Infancia y Políticas Públicas (2012) por la Universidad de la República (UR). Docente e investigadora del Instituto de Psicología de la Salud de la Facultad de Psicología (UR). Trabaja en líneas de investigación vinculadas a género, violencia y sistemas penales juveniles. Orcid: 0000-0002-8279-9877. Índice H5: 10. Correo electrónico: galeotti@psico.edu.uy

**** Licenciada en Psicología (2010) por la Facultad de Psicología de la Universidad de la República. Magíster en Psicología Social (2015) por la Facultad de Psicología (UR). Candidata a doctora en Estudios de género: culturas, sociedades y políticas por la Universidad Autónoma de Barcelona. Docente e investigadora del Instituto de Psicología Social de la Facultad de Psicología, (UR). Investigadora activa del Sistema Nacional de Investigadores de la Agencia Nacional de Investigación e Innovación (Anii). Líneas de investigación: género, cuidados y sistemas penales juveniles. Orcid: 0000-0002-2516-4488. Índice H5: 1. Correo electrónico: cmontes@psico.edu.uy 


\section{Managing sexualities in criminal systems: female adolescents}

- Abstract (descriptive): This paper discusses the management offemale adolescents'sexualities in the Juvenile Criminal System in Uruguay. The study uses a qualitative research perspective that identifies the production of meanings by key agents, supported by the contributions of feminist epistemologies and criminologies. The authors discuss two analytical tensions, one related to the sexualization of the body and its overlapping with sexuality, and another based on the treatment of adolescent motherhood linked to sin and the exercising of sexuality. Finally, we show how many of these interventions target the body through the establishment of socio-criminal control practices aimed at the female adolescents' sexualities.

Key words: Juvenile delinquency, adolescent women, sexuality (Social Sciences Unesco Thesaurus).

\section{Gestão das sexualidades nos sistemas penais: as adolescentes mulheres.}

- Resumo (descritivo): o artigo reflexiona sobre a gestão das sexualidades das mulheres adolescentes tomando como ponto de partida o Sistema Penal Juvenil Uruguaio. Trabalhamos com uma perspectiva de pesquisa qualitativa que se concentra na produção de significados pelos principais agentes, aos quais adicionamos as contribuições de epistemologias e criminologias feministas. Apresentamos duas tensões analíticas, uma relacionada à sexualização do corpo e à subjugação da sexualidade. E outro sobre o tratamento da maternidade adolescente ligada ao pecado e ao exercício da sexualidade. Finalmente, mostramos quantas intervenções são sustentadas no corpo, ao configurar práticas de controle sócio-criminal voltadas para a sexualidade das mulheres adolescentes.

Palavras-chave: delinquência juvenil, adolescentes mulheres, sexualidade (Thesaurus de Ciências Sociais da Unesco).

-1. Introducción: acerca de la particularización de las adolescentes mujeres y su vínculo con los sistemas penales. -2. Contextualización socio-histórica. De claustros y tutelas en el Uruguay del siglo XX. -3. Itinerarios metodológicos. -4. Los aportes de las Epistemologías y las Criminologías Feministas para pensar el género de los sistemas penales juveniles. $\mathbf{- 5}$. Resultados de análisis: la gestión de las sexualidades en adolescentes mujeres en los sistemas penales. -Lista de referencias.

\section{Introducción: acerca de la particularización de las adolescentes mujeres y su vínculo con los sistemas penales}

En el presente artículo abordamos la gestión de las sexualidades en los sistemas penales juveniles, en especial el uruguayo (GaleottiGalmés 2013; González, 2015; González, Leopold, López-Gallego, \& Martinis, 2013; Leopold, 2014; López-Gallego, 2016; Morás, 1992) e intentamos discutir algunas dimensiones claves de las prácticas de control socio-penal dirigidas al disciplinamiento del cuerpo y en especial aquello del cuerpo que se vincula al ejercicio de la sexualidad en adolescentes mujeres (Abal, Cheroni, \& Leopold, 2005;
Pitch, 2003). En este sentido, cabe considerar algunas particularidades de las adolescentes que transitan por los sistemas penales juveniles. Reguillo (2000) señala que la "edad" no se agota en cuestiones de orden biológico sino que asume singularidades vinculadas a las posiciones sociales. Esto permite pensar la adolescencia como una construcción social, inscripta en un tiempo-espacio específico, el cual es necesario problematizar para poder pensar las particularidades de ser mujer, adolescente y transitar por los sistemas penales.

Por su parte Fernández (1993) señala que el proceso de particularización de la infancia y adolescencia, invisibiliza dos inscripciones fundamentales: la clase social y las relaciones entre los géneros. Las vulnerabilidades de las 
niñas convertidas en adolescentes de las clases pobres estarán signadas por la desprotección sexual y la explotación laboral. Los trabajos domésticos y/o de cuidados, las maternidades forzadas y la explotación sexual comercial serán algunas de la estrategias biopolíticas que configurarán el pasaje por la adolescencia de estas mujeres.

Utilizamos la noción de prácticas de control socio-penal, para reflexionar en términos de un campo de problemas heterogéneo compuesto por una multiplicidad de vectores, que nos hablan de las formas que tienen las personas de ser juzgadas $\mathrm{y} / \mathrm{o}$ tratadas en función de sus desvíos a la normativa penal imperante en un determinado contexto socio-histórico. Configuran prácticas sociales concretas en términos de daño, responsabilidad, castigo y/o pena, que "creo son algunas de las formas empleadas por nuestra sociedad para definir tipos de subjetividad, formas de saber y, en consecuencia, relaciones entre el hombre y la verdad que merecen ser estudiadas" (Foucault, 1978/1991, p. 17).

En la presente reflexión buscamos profundizar e interrelacionar aportes teóricos con los resultados de investigaciones realizadas en el sistema penal juvenil uruguayo, en el marco de las epistemologías feministas (Alcoff \& Potter, 1993; Haraway, 1995; Harding, 1987, 1996; Nicolás-Lazo, 2009) y las criminologías críticas feministas (Almeda, 2017; Larrauri, 1994; Miller, 2003; Smart, 1976) para mostrar la gestión de la sexualidad de las adolescentes mujeres por medio de la sexualización de las conductas y del cuerpo de las jóvenes y el tratamiento de la maternidad asociado al pecado.

El texto se subdivide en cuatro apartados, en el primero de ellos "Introducción: Acerca de la particularización de las adolescentes mujeres y su vínculo con los sistemas penales" planteamos el campo de problemas que vincula a las adolescentes mujeres con los sistemas penales juveniles. En el segundo denominado "Contextualización socio-histórica. De claustros y tutelas en el Uruguay del siglo XX”, presentamos una perspectiva genealógica acerca de las prácticas de control socio-penal en las adolescentes mujeres en el Uruguay del siglo XX. En el tercer apartado "Itinerarios metodológicos", discutimos los caminos seguidos para la producción de la información desde una perspectiva de investigación cualitativa. En el apartado cuarto "Los aportes de las epistemologías y las criminologías feministas para pensar el género de los sistemas penales juveniles" trabajamos en torno a dichos aportes teóricos para comprender las relaciones de poder que construyen los sistemas sexo-género en el contexto de los sistemas penales juveniles. Y por último, en el apartado "Resultados de análisis: La gestión de la sexualidad en adolescentes mujeres en los sistemas penales" utilizamos los aportes esbozados para presentar dos líneas analíticas enmarcadas como tensiones: De la sexualización del cuerpo al avasallamiento de la sexualidad y el tratamiento de la maternidad adolescente vinculado al pecado y el ejercicio de la sexualidad.

\section{Contextualización socio-histórica. De claustros y tutelas en el Uruguay del siglo XX}

En el transcurso del siglo XX, se configuran determinadas particularidades en el abordaje socio-penal de las niñas y adolescentes mujeres. El modelo tutelar, caracterizado por niños/as que peligran de convertirse en peligrosos (Donzelot, 1977/2008) configura el debate acerca de un derecho penal de autor en el que el sistema penal juvenil cumple la función de examinar personas más que delitos, asesorado por los saberes médicos, psicológicos, sociológicos y psicopedagógicos.

La noción de menor se convierte en una subcategoría de la infancia pobre, en principio vinculada a los excedentes de la escuela, luego entrarán otros sujetos en función de otras vulnerabilidades sociales (Leopold, 2014). En relación a las menores mujeres, la intervención de las instituciones religiosas le impondrá una cuota mayor de tutela y control. La instauración de las "casa correccionales" en Latinoamérica, a través de la Congregación del Buen Pastor, signa la tendencia conventual y de claustro para el tratamiento de las "mujeres desviadas". Las prácticas de control socio-penal se caracterizan 
por la capacitación de las mujeres para la vida doméstica y/o la servidumbre, lo que supone la desvinculación de las niñas y adolescentes del contexto familiar, y la atribución de una potencial unión entre pobreza, abandono, delito y pecado (Aguirre, 2008; Galeotti-Galmés, 2013; Juliano, 2009).

En el caso de Uruguay, el asilo del Buen Pastor se inauguró en 1876, en la ciudad de Montevideo, para albergar a niñas, adolescentes y mujeres jóvenes desviadas, sobre todo pobres, la reforma moral del alma de las niñas daba comienzo. Si bien en el Uruguay del siglo XX se asiste a un proceso de secularización que establece la laicidad para todos los establecimientos públicos, las mujeres institucionalizadas son su excepción (adultas y menores de edad) al ser objeto de una respuesta tutelar híbrida: la institucionalidad estatal y la actividad privada de carácter religioso.

La investigación realizada en el año 1946, por la psicóloga y maestra Reyes, es uno de los pocos testimonios escritos que da cuenta de las prácticas de control socio-penal que se realizaban con mujeres en el Hogar Femenino No.1 del Consejo del Niño, en la ciudad de Montevideo. Reyes refiere a la importancia que tiene el cuerpo vinculado a la sexualidad de las adolescentes en la reforma y el disciplinamiento de las que son objeto las adolescentes en el Asilo del Buen Pastor. Al referirse a los motivos por los cuales las conductas sexuales tienen un lugar tan protagónico, Reyes arriesga una explicación psicoanalítica, vinculada a la represión de los propios instintos sexuales que deben de llevar a cabo las religiosas (voto de castidad). "Cabe observar que la aplicación de castigos satisface las tendencias agresivas de quienes los aplican, fortaleciéndose la inhibición de conductas prohibidas pero deseadas inconscientemente (...)" (Reyes, 1989, p. 22).

La Orden del Buen Pastor fortalece el discurso tutelar de la época, que aboga por un derecho penal de actor más que de acto. Las estrategias y tecnologías focalizan en la reforma moral de las adolescentes, a través de la asimilación de delito con pecado, el refuerzo de los modelos femeninos de la mujer-madre, mujer-ama de casa y mujerservidumbre doméstica y la fuerte disciplina que recae en el ejercicio de la sexualidad de las niñas y adolescentes (López-Gallego, 2016).
En este contexto, los motivos de ingreso a dicha organización así como a la institución estatal propiamente dicha (Consejo del Niño) se fundamentan en criterios morales de índole sexual. La significación de mala conducta aparece sustentada a determinado ejercicio de la sexualidad y de las relaciones amorosas de las adolescentes, siendo vía de entrada a la institucionalización solicitada indistintamente por cuidadores y/o padres (Aguirre, 2008; Portillo, 1989). El número de niñas $\mathrm{y}$ adolescentes ingresadas por delitos tipificados en el Código Penal era insignificante, lo que supone una práctica alejada de las concepciones del derecho penal moderno, que sí eran aplicadas en la justicia de varones (Fessler, 1998). En este sentido, las acciones privilegiadas en este híbrido institucional recaen en la formación de estas adolescentes para la vida doméstica mediante mecanismos de "colocación familiar" (con o sin salario) como vía de posible egreso de la institucionalización valorando como atributos necesarios la docilidad, honradez e higiene personal (Fessler, 1998).

En las investigaciones históricas realizadas por Almeda (2002), acerca de la privación e institucionalización de mujeres en España, surge como constante la fijación en el cuerpo y el control de la práctica de la prostitución ejercido hacia las mujeres. Almeda (2002) expresa que es el binomio prostitución-pobreza el elegido por el control socio-penal, dado que las "cortesanas" de la época reciben otro tipo de tratamiento. El trabajo doméstico, las enseñanzas religiosas constantes y la sumisión a las firmes normas de la institución, son las tecnologías elegidas para "domesticar a la fiera" (...) según las normas de la época: ser una perfecta esposa o dedicarse a las tareas de servir (Almeda, 2002, p. 36). Con las mujeres adolescentes, es fundamental separarlas y clasificarlas en tanto que pecadoras, para así prevenir a posibles hombres "descuidados" que pueden ser atraídos por sus encantos físicos. Nuevamente, el cuerpo, la sexualidad y la explotación sexual comercial ocupan la preocupación principal.

(...) hay muchas mujeres mozas vagabundas y ociosas, y entre ellas algunas muchachas de dieciséis y menos años, que no se sustentan de otra cosa, sino del mal vivir. Para esto, llegada la 
noche, salen como bestias fieras de sus cuevas a buscar la caza. Pónense por esos cantones, por calles y portales de casas, convidando a los miserables hombres que van descuidados $\mathrm{y}$, hechas lazos de Satanás, caen y hacen caer en gravísimos pecados (De San Jerónimo, 1608, citado por Almeda, 2002, p. 31).

\section{Itinerarios metodológicos}

Los itinerarios metodológicos que trazamos implican la comprensión de situaciones particulares y únicas-los funcionamientos de las medidas judiciales destinadas a adolescentes mujeres- en un contexto institucional específico y/o escenario donde se ejecutan las medidas judiciales -SPJU- a través de los sentidos que les conceden los/las agentes -adolescentes, técnicos/as, autoridades, investigadores/asque allí viven o trabajan. De manera amplia, situamos estos itinerarios en el gran campo de las metodologías cualitativas de investigación y siguiendo a Denzin y Lincoln (2003) las entendemos como:

Una actividad que localiza al observador en el mundo. Consiste en un conjunto de prácticas interpretativas que hacen al mundovisible. Estas prácticas transforman el mundo, lo convierten en una serie de representaciones, que incluyen las notas de campo, las entrevistas, conversaciones, fotografías, registros y memorias. En este nivel, la investigación cualitativa implica una aproximación interpretativa y naturalista del mundo. Esto significa que los investigadores cualitativos estudian la cosas en su contexto natural, intentando dar sentido o interpretar los fenómenos en función de los significados que las personas le dan (p. 3).

La muestra intencional (Ritchie \& Lewis, 2003), característica de lo cualitativo, se compone de dos espacios institucionales, donde el criterio que opera es la variedad en términos de regímenes de sanción, encierro versus libertad. En régimen de encierro, existe una única dependencia para todo el Uruguay: el Centro de Ingreso de Adolescentes Femeninos (Ciaf). Para las medidas alternativas al encierro se elige el Programa de Medidas Socioeducativas No Privativas de Libertad y Mediación (Promesem), único programa dependiente del Instituto Nacional de Inclusión Social Adolescente (Inisa), institución rectora en materia de ejecución de sanciones del SPJU.

La investigación etnográfica en las instituciones seleccionadas (Bryman, 2001; Hammersley, 2007) nos permite atender a la producción de sentidos de los diversos actores/ actrices claves que participan de la ejecución de las medidas judiciales en adolescentes mujeres. Se recurre a diversas técnicas de investigación que incluyen la observación de los diferentes espacios físicos y de las interacciones que allí se producen, conversaciones informales con los/as distintos/as trabajadores/as, entrevistas en profundidad con las autoridades del lugar y operadores/as técnicos/as, entrevistas en profundidad con las adolescentes y la realización de cuatro acompañamientos de adolescentes mujeres en sus tránsitos por las medidas no privativas de libertad. Realizamos un exhaustivo registro en un Diario de Campo que da cuenta de las diversas actividades realizadas.

\section{Los aportes de las epistemologías y criminologías feministas para pensar el género de los sistemas penales juveniles}

Pitch (2003) establece que la autonomía para las mujeres está relacionada al dominio de su cuerpo, un cuerpo históricamente sometido al dominio público desde diversos discursos: médicos, jurídicos, políticos, pedagógicos, entre otros. El cuerpo construido como femenino es así postulado como un espacio público para el Derecho, en tanto que su discurso habla de sólo un cuerpo que requiere ser normatizado, el cuerpo de las mujeres, mientras que el cuerpo masculino es el estándar, la norma (Ferrajoli, 2003).

La libertad femenina es, directa o indirectamente una libertad del cuerpo, y precisamente de un cuerpo sexuado, de un cuerpo femenino: bien se trate de la libertad sexual, es decir de la inmunidad del cuerpo de la mujer frente a violaciones, abusos sexuales, acoso, mutilaciones genitales o servidumbres conyugales; bien se trate de autodeterminación de la maternidad y de sus formas, y en 
cualquier caso de no constricción a embarazos forzados; bien se trate de inmunidad frente a la prostitución forzada, o de explotación de la prostitución o de tráfico de personas, o también de protección paternalista impuesta a las prostitutas, bien se trate, en general, de libertad como autodeterminación del propio futuro vital y laboral (Ferrajoli citado por Pitch, 2003, p. $13)$.

En estos marcos, se vuelve prioritario situar los sistemas sexo-género (Rubin, 1975) como principios organizadores de las prácticas y discursos de los sistemas penales. Las prácticas de control socio-penal dirigidas a adolescentes mujeres en el sistema penal juvenil uruguayo (SPJU), las pensamos como tecnologías de género, en tanto fijan y producen cuerpos generizados (Butler, 2002; de Laurentis, 1989; Miller, 2003).

La noción de sistema sexo-género es una forma de ampliar el campo discursivo del género, más allá de resabios binarios y pensando ambas nociones en forma articulada y relacional. Ubicamos la noción de género como una categoría de análisis (Scott, 1996) de lo social en intersección (Crenshay, 2004) con otras categorías como edad, clase social o raza. El género en función de su capacidad performativa, de improvisación que se construye con otros y para otros (Butler, 2006). Butler (2001) pone en tensión la continuidad entre sexo y género, donde el género es postulado en términos de performance y la diferencia sexo-genérica es pensada en un contexto de relaciones de poder y producción de efectos de un "sexo prediscursivo". En este sentido, la posición dual biología-cultura es una expresión más de las lógicas binarias que construyen ordenamientos jerárquicos.

Así critica el esencialismo que rodea las teorizaciones relativas al género, en las que la utilización de estrategias epistemológicas colonizadoras anulan la relevancia de la diversidad de contextos culturales. El desafío es pensar de otro modo y poder incluir criterios multirreferenciales como raza, clase social, desde una dimensión socio-histórica que permita situar las prácticas sociales en su vertiente genealógica (López-Gallego, 2011). Daly (2013) subraya algunas contribuciones que las teorías feministas aportan a la criminología; el modo "clase-raza-género" insiste en considerar a las personas en una matriz de múltiples relaciones sociales, el modo "haciendo género" pone el acento en los escenarios de interacción social.

En función de lo planteado nos situamos en una noción de género que no se encuentra estrechamente ligada a la diferencia sexual, sino como producto de múltiples tecnologías sociales, donde los dispositivos propuestos por los sistemas penales juveniles serán uno de los espacios elegidos para tensionarla. Comprendemos al sistema sexo-género como "una construcción sociocultural, un aparato semiótico, un sistema de representación que asigna significado (identidad, valor, prestigio, ubicación en la jerarquía social, etc.) a los individuos en la sociedad" (de Laurentis, 1989, p. 11). Con el género de los sistemas penales juveniles introducimos una dimensión de reflexión que pretende transversalizar los sistemas sexo-género en las producciones criminológicas. Desde las epistemologías feministas se resalta el carácter político del conocimiento y del sistema sexo-género como uno de los posibles posicionamientos que permean la producción de lo científico.

Diversas investigaciones en el marco de las criminologías críticas (Birgin, 2000a, 2000b; Bodelón, 2003; Brown, Chesney-Lind, \& Stein, 2007; Carlen \& Worrall, 2004; Pitch, 2003; Smart, 2000) integran las relaciones de género como una dimensión presente en los sistemas penales y como un elemento fundamental para analizar la posición desigual de las mujeres. Los sistemas penales son analizados como dispositivos útiles para la dominación y subordinación de las mujeres. Estos estudios analizan el fenómeno, mostrando cómo el Derecho dentro de sus credenciales tiene potenciales creadores de tecnologías para la construcción de los sistemas sexo-género.

Smart (2000) explica y analiza al Derecho en tres dimensiones: una que muestra la ley escrita la cual puede ser interpretada en diversos modos; la segunda vinculada con la utilización de la ley a través de los agentes legales en sus prácticas cotidianas y la tercera tiene que ver con la capacidad del derecho de crear y recrear 
subjetividades. En este sentido, plantea que el Derecho es también una forma de sexuación y generización de los cuerpos. Así colabora en la construcción del género que además define los sexos y les atribuye por consiguiente unas sexualidades (Butler, 2006; Smart, 2000). A lo largo de la historia el rol del Derecho simbólico perpetúa las relaciones de subordinación de las mujeres en concordancia con la producción de formas de dominación que invisibilizan y las dispone como objetos del derecho (Fries \& Matus, 2000).

Estos análisis muestran cómo el derecho y los subsiguientes sistemas penales se construyen sobre universales -masculinizadosy naturalizados como únicos esquemas, de forma normativa. Los análisis contemporáneos permiten cuestionar estas normalizaciones y visualizar que no hay sentidos únicos sino tensiones (Pitch, 2003).

En las referencias a la incidencia de la criminalidad de las mujeres se maneja la hipótesis de que los sistemas penales son más benevolentes con las mujeres que los varones justificando con argumentos como el número menor de mujeres encarceladas o detenidas por ejemplo. Estudios en el tema (Carlen \& Worrall, 2004; Chesney-Lind, 1989; Larrauri, 1994) indican que las mujeres que transgreden la ley viven un doble castigo. Por un lado por la transgresión a las normas penales y por otro por transgredir las normas de género y los roles sociales asumidos y asignados (Carlen, \& Worrall, 2004). En este marco se constituyen los controles domésticos en el ámbito de las relaciones de proximidad y familiares, los controles médicos (medicalización y psiquiatrización como forma de resolver los conflictos o acallarlos) y el control de las apariciones y actividad en la vida pública (Larrandart, 2000; Larrauri, 1994).

De esta manera y como muestran Carlen y Worrall (2004) las mujeres seleccionadas por los sistemas penales muestran la doble desviación: a la normatividad penal y de género y sobre ellas recae un triple disciplinamiento que obedece a procesos de medicalización, feminización y domesticación.

En primer lugar, la medicalización a través de la sedación que se produce con el consumo de psicofármacos parece ser un tratamiento mayoritario de las adolescentes que transitan por el SPJU. Los sentidos que le adjudican las adolescentes y los operadores técnicos a estas prescripciones médicas son diversos; sustitución en adicciones previas, paliativo de los efectos nocivos del encierro como insomnio y angustia, mecanismo de contención física que evita los desbordes agresivos, mecanismo paliativo del tiempo rutinario y ritualizado, entre otras.

Algunas investigadoras (Antony, 2007; Azaola, 2007; Larrandart, 2000) sostienen que el uso de medicación psiquiátrica es más común en mujeres que en varones. Organizaciones dedicadas a la protección de los derechos humanos (Comité de los Derechos del Niño, 2015; Institución Nacional de Derechos Humanos y Defensoría del Pueblo de la República Oriental del Uruguay, 2015) han efectuado recomendaciones que tienen que ver con: dejar registrado en la historia clínica el uso de la medicación y el porqué de su indicación en términos de un diagnóstico preciso, revisar la misma cada treinta días y que sólo pueda ser prescrita por el médico tratante.

En segundo lugar, la domesticidad es una dimensión prioritaria de la particularización de las adolescentes mujeres. Así, cobra fuerza la conceptualización de los sistemas penales como tecnologías de género que fijan sentidos hegemónicos acerca de lo que es "ser mujer". Las propuestas formativas, en muchos casos, tienen un claro sesgo de género y de clase social, lo que tristemente coincide con muchas de las expectativas de las adolescentes que se proyectan como buenas madres, esposas y/o empleadas domésticas. Ejemplo de esto es la gran dedicación de tiempo que las mujeres adultas y las adolescentes dedican al cuidado de personas dependientes y tareas de limpieza y sostenimiento del hogar (López-Gallego \& Montes-Maldonado, 2016). En Uruguay hay investigaciones que afirman que son las mujeres jóvenes y adolescentes mayormente de sectores empobrecidos de la sociedad quienes cuidan de niños/as pequeños/as y adultos mayores y se ocupan de las tareas domésticas. Esta alta dedicación horaria dos veces y media más en mujeres que en varones hace que estas 
se alejen o tengan una presencia intermitente en el sistema educativo y el mercado laboral (Katzkowicz et al., 2015).

En los sistemas penales juveniles las mujeres viven un proceso de domesticación y transitan por medidas judiciales (denominadas socio-educativas) que buscan moldearlas para un determinado ejercicio de la vida familiar. Las tareas de cuidado no son visualizadas como una importante carga de trabajo que le genera limitaciones para desarrollarse en otras áreas de la vida sino realizadas a partir de un rol de género que las coloca y ubica en el lugar de cuidadoras mediante unos ideales de cuidado (Hochschild, 2003/2011) tradicionales (LópezGallego \& Montes-Maldonado, 2016).

Finalmente, la maternidad es una constante preocupación el SPJU, sobre todo en el espacio de reclusión de mujeres. Principalmente porque en la mayoría de las sociedades son las mujeres las que se encargan de las tareas de cuidado de sus hijos/as y, entre otras cosas, hay que gestionar el tema de los niños/as que viven con sus madres durante la privación de libertad. A esto se suma la cuestión de la adolescencia, la maternidad en las adolescentes es un tema recurrente de alerta social, lo que determina que se convierta en un espacio privilegiado de control de la sexualidad y el cuerpo de las mujeres menores de edad.

Estudios en cárceles (Antony, 2007; Igareda, 2009; Lagarde, 2014) concluyen que el tratamiento carcelario refuerza la ecuación de mujer $=$ madre en términos esenciales, como mandato vinculado a la feminidad. De este modo, se fijan sentidos acerca de lo que implica ser buena o mala madre, siendo la ecuación mala madre $=$ criminal un elemento a problematizar con los agentes institucionales. Está muy presente la valoración moral en términos de "buenas o malas madres", en la medida que la transgresión a los mandatos de género comparta sanciones que excede la normativa penal.

Los sistemas penales juveniles producen tecnologías de género por medio de la fijación de significados en torno a las diferencias de género. En este artículo, nos preguntamos acerca del despliegue de técnicas y dispositivos específicos para el tratamiento de las sexualidades de las mujeres adolescentes en los sistemas penales y cómo los mismos operan en la construcción de los sistemas sexo-género. Algunas claves hemos introducido en relación a los procesos de domesticación, feminización y medicalización, la apuesta en los resultados de análisis es discutir acerca de la gestión de las sexualidades en los sistemas penales juveniles, específicamente el caso de las adolescentes mujeres.

\section{Resultados de análisis: La gestión de las sexualidades en adolescentes mujeres en los sistemas penales.}

En términos históricos las mujeres han sido objeto de tutelas, sin plena responsabilidad a lo largo del siglo XX; siendo el cuerpo, más específicamente la sexualidad el espacio privilegiado del control socio-penal. Algunas de estas prácticas de control conllevan sanciones legales -aborto ${ }^{1}$ y prostitución-. Los sistemas penales producen relaciones de dominación a través de determinados mecanismos que fijan y reproducen determinadas identidades del ser mujer de manera inflexible y unívoca. En este sentido, Bodelón (2003) advertirá que los sistemas penales construyen relaciones de subordinación, tanto a través de la discriminación como de la igualación. Las reivindicaciones feministas de una justicia igual en el plano formal no significan necesariamente igualdad, dado que las normas son aplicadas a personas con profundas desigualdades sociales. "Las mujeres criminalizadas deben enfrentar prácticas jurisdiccionales e institucionales profundamente marcadas por las relaciones patriarcales" (Bodelón, 2003, p. 454).

Si antes hacíamos mención a que los sistemas penales históricamente sitúan a las mujeres en la misma categoría que la niñez, ¿qué sucede cuándo se comparte la imprecisa categorización de ser un poco mujer, un poco niña? Doble condición que torna a las adolescentes más vulnerables a la fijación

En Uruguay recientemente se ha despenalizado el aborto a través de la Ley No. 18.987 (Interrupción voluntaria del embarazo). Disponible en http://www.parlamento.gub.uy/leyes/ AccesoTextoLey.asp?Ley=18987\&Anchor $=$ 
de determinadas posiciones sobre las que el sistema sexo-género cobra un papel central.

En este sentido se realiza una atribución de significados del manejo y manifestación del cuerpo y sexualidad que las desdibuja de su condición adolescente, al tiempo que reproduce desde visiones patriarcales y de dominio adultocéntrico desigualdades de género y edad.

\subsection{Tensión primera: De la sexualización del cuerpo femenino al avasallamiento de la sexualidad}

En esta primera tensión introducimos la noción de sexualización para referirnos a cómo muchas de las dimensiones de la vida de las adolescentes mujeres en sus tránsitos por los sistemas penales son entendidos en clave sexual, con la contracara de que en muchas ocasiones otras dimensiones de las sexualidades, como ser las violencias sexuales, son negadas y avasalladas.

Frecuentemente durante el proceso se hace referencia a la actividad sexual de las chicas. Esta información se utiliza de tal forma que se produce lo que se ha denominado un proceso de "sexualización de la delincuencia femenina". Es decir, frecuentemente los/as profesionales consideran que las chicas con problemas tienen problemas con su sexualidad (Bodelón \& Aedo, 2015, p. 228).

Almeda (2002) sostiene que uno de los principios de rehabilitación de las prácticas penales -especialmente con mujeres encarceladas- apunta a lo que denomina reconstrucción de la domesticidad perdida. En este sentido establece el reforzamiento de éstas al mundo privado del hogar y a las tareas propias del mismo. En el caso particular de las prácticas del sistema penal juvenil con adolescentes mujeres esta afirmación adquiere relevancia determinada no sólo por condiciones de género sino también por la edad, y en la cual se tiende a considerar con naturalidad su correspondencia a la vida familiar regidas por la autoridad de los padres.

Dentro de esta estrategia de domesticidad se ha señalado el abordaje técnico y profesional de los cuerpos de las adolescentes en términos de su sexualidad y ejercicio de la misma. La percepción que se tiene de sus apariencias físicas, actitudes y conductas las colocan alejadas de los cánones sociales esperados de lo femenino y por tanto- en términos dicotómicos de división de los géneros- con atributos considerados masculinos (Galeotti-Galmés, 2013). Esta masculinización, entendida desde posiciones subjetivas de actividad y circulación pública, así como supuestos de ejercicio activo de su sexualidad, constituye un punto de partida para intervenciones técnicas basadas en el modelado disciplinante a través del enunciado autocuidado consistente en el cuidado estético y de belleza sumado a otros aspectos de salud referidos específicamente al cuidado y control del ejercicio sexual. Este aspecto supone para los/as operadores/as del sistema penal juvenil un diferencial respecto a los adolescentes varones en una doble vertiente, la concepción de sexualidad en adolescentes, en tanto visualizada desde parámetros adultocéntricos asociada a problema y "peligrosidad" (LópezGómez, 2005, p. 27) y por otro lado aquello significado en el cuerpo como rebelde, fuera de lugar o impropio (Galeotti-Galmés, 2013).

Ambos aspectos -reintegro al mundo familiar/paternal y disciplinamiento de la sexualidad- presentan una cuestión paradojal en el tratamiento socio penal de estas adolescentes. Diversas investigaciones señalan que en la mayoría sus historias de vida se encuentran signadas por situaciones de abuso sexual familiar y en forma concomitante vulnerables a diversos escenarios de explotación sexual comercial y no comercial (Acale-Sánchez, 2017; Chesney-Lind, 2013; Pasko \& López, 2015).

El abordaje institucional oscila entre la sexualización, es decir, el conferir carácter sexual a casi cualquier cuestión o comportamiento de las adolescentes y la negación de la sexualidad de las jóvenes, como estrategia para controlar una sexualidad que se piensa como descontrolada. Uno de los temas recurrentes vinculados a la sexualidad femenina en los sistemas penales juveniles es la prostitución, o mejor denominado explotación sexual comercial y no comercial; situación que históricamente ha sido vista como sancionadora y con estatus delictual. Delito y/o pecado en el que se solapa la impronta moralista-religiosa que ha teñido el tratamiento penitenciario de las 
mujeres. "Para las mujeres, lo que se considera pecado era básicamente la utilización autónoma de su sexualidad" (Juliano, 2009, p. 82).

Esta oscilación invisibiliza que algunos procesos de criminalidad se encuentran precedidos por actos de victimización y por tanto "lo que puede ser una estrategia de supervivencia es criminalizada" (Pasko, 2013, p. 29). La experticia de distintos/as operadores/ as técnicos/as que trabajan con adolescentes mujeres infractoras indican la presencia recurrente de victimizaciones tempranas de violencia sexual, las cuales no son incluidas como componentes diferenciales del abordaje que se realiza. En este sentido se trabaja a modo de denegación -se reconoce pero se desconoce en el diseño de la intervenciónuna problemática que muchas veces tiene incidencia directa en la situación delictual por la cual fue captada por el sistema penal juvenil. Mecanismo de denegación que aparece sustentado en aspectos representacionales del/a técnico/a sobre sexualidad, violencia sexual y adolescencia, y el desconocimiento de sus efectos (Galeotti-Galmés, 2013). En este punto la estrategia de reintegro a su familia, sin condiciones de problematización causal de las situaciones de violencia sexual vividas señala su paradoja, con efectos revictimizantes y de pérdida de singularidad subjetiva.

Por otra parte, el hecho de ser menores de edad lleva a que no se piense en las visitas conyugales en contextos de privación de libertad, situación que deja a las adolescentes en una clara desventaja frente a sus pares adultos en relación al ejercicio de su sexualidad. De esta forma, la sexualidad es valorada por los operadores/as técnicos/as y jurídicos en dos sentidos: por una parte es la medición utilizada para diagnosticar la adecuación de las adolescentes a los códigos sociales imperantes y, por otra parte, es evaluada como incontrolable e irresponsable, algo de lo que hay que estar siempre alerta.

\subsection{Tensión Segunda: El tratamiento de la maternidad de las adolescente vinculado al pecado y el ejercicio de la sexualidad}

Evidentemente asociado al tratamiento de la sexualidad se encuentran las cuestiones de fecundidad, embarazo y maternidad.
Estudios en el campo de la fecundidad en adolescentes en Uruguay (López-Gómez \& Varela, 2016; Varela \& Lara, 2015) dan cuenta del descenso del nivel de fecundidad total en los últimos años contrariamente a lo que sucede con el comportamiento reproductivo de las adolescentes donde se produce un incremento de la tasa de fecundidad ubicándose actualmente cerca de 62 por mil con una tendencia al estancamiento y sin rasgos de modificarse. Este estancamiento se puede explicar mediante el relacionamiento de este fenómeno con condiciones estructurales como ser: bajos niveles educativos, desigualdades de género, barreras para el acceso a los derechos de salud sexual y reproductiva, entre otras. Estas cifras ubican a Uruguay en una alta tasa de fecundidad y maternidad en adolescentes en comparación con el mundo y en torno al promedio de América Latina.

Este aspecto aparece agravado en situaciones de embarazo y maternidad en niñas menores de 14 años. A diferencia de lo que ocurre en la franja de adolescentes de entre 15 y 19 años donde se registra incidencia de embarazos por iniciación sexual temprana, la mayoría de los casos de embarazo en menores de 14 años son producto de violencia sexual ejercida por familiares, vecinos $\mathrm{y} / \mathrm{o}$ desconocidos. Constituyen embarazos y maternidades forzadas en un contexto de vulneración de derechos por causales de parejas y/o matrimonios forzados, falta de acceso a servicios de salud, deserción escolar y presiones familiares (Balance Regional Cladem, 2016).

Esta realidad también se visualiza en el SPJU y por ende allí se producen estrategias para abordar los problemas que le suscita la fecundidad en las adolescentes. Así se realizan fuertes controles ginecológicos, prevención del embarazo, medicalización, constituyendo aquello que Pitch (2009) denomina la prevención sexuada donde las mujeres cobran un lugar central (prevención o cuidado de embarazos, enfermedades de transmisión sexual, controles ginecológicos periódicos, entre otras prácticas de prevención y/o promoción de salud) y se ubica como una de las modalidades de control social, en este caso, feminizados. Esta 
modalidad de control se encuentra potenciado en los sistemas penales donde las adolescentes se encuentran en situación de encierro, fuertemente vigiladas y se ve transversalizado además por la moral socio sexual (Hernández, 2013) del personal técnico, educativo o responsables de la ejecución de las medidas de castigo. Cabe destacar que en los sistemas penales se producen controles sobre aspectos de la vida que antes las adolescentes mantenían como aspectos privados o íntimos. Es decir, ahora todas sus actividades son realizadas bajo la mirada de otros: llamadas telefónicas, visitas, actividades educativas, vínculos sociales en general o con otras adolescentes dentro de los centros, planificación de proyecto de vida, etc.

El abordaje de la fecundidad y maternidad en las adolescentes se constituye también como una herramienta de restitución de la domesticidad pérdida (Almeda, 2002) o lógica de domesticidad (Ballesteros, 2017) donde el sistema utiliza los ideales de maternidad y cuidado para controlar a las mujeres. Estos elementos que demuestran la presencia de la moralidad, ideales religiosos e intentos por transmitir determinados ideales heteronormativos de feminidad han sido ejemplificados en otros estudios del tratamiento de las mujeres en los sistemas penales (Almeda, 2002, 2017; Ballesteros, 2017; Chesney-Lind \& Pasko, 2013, de Miguel, 2017).

Vigilanciay moral socio sexual, atribuciones negativizadas del comportamiento sexual desde significados construidos a partir de parámetros de masculinización y denegación de las historias de violencia sexual que sufren las adolescentes que transitan por medidas judiciales constituyen una modalidad que particulariza su tratamiento y miradas en el abordaje. En relación a esto, las prácticas socio penales con estas adolescentes aparecen sustentadas desde lógicas alejadas de una perspectiva de comprensión de los aspectos que la atraviesan en términos de género y de derechos, contraviniendo concepciones actuales sobre infracción adolescente. La forma en que se enfatiza en la sexualidad y sus manifestaciones, de las adolescentes mujeres que cometen una infracción a la ley penal, compone una de las paradojas del sistema penal juvenil en tanto acarrea lógicas tutelares de antaño al mismo tiempo que reproduce el estatus religioso que en su principio las origina.

\section{Lista de referencias}

Abal, A.; Cheroni, A., \& Leopold, S. (2005). Adolescencia e infracción: una aproximación a la construcción subjetiva. Montevideo: Inau, Cenfores.

Acale-Sánchez, M. (2017). El género como factor condicionante de la victimización y de la criminalidad femenina. Papers, 102(2), 231-259. doi:http://dx.doi. org/10.5565/rev/papers. 2334 .

Aguirre, C. (2008). Denle duro que no siente: poder y transgresión en el Perú republicano. Lima: Pedagógico San Marcos.

Alcoff, L. \& Potter, E. (1993). Feminist Epistemologies. Nueva York: Routledge.

Almeda, E. (2002). Corregir y castigar: el ayer y hoy de las cárceles de mujeres. Barcelona: Bellaterra.

Almeda, E. (2017). Criminologías feministas, investigación y cárceles de mujeres en España.Papers, 102(2), 151-181.doi:http:// dx.doi.org/10.5565/rev/papers.2334.

Antony, C. (2007). Mujeres invisibles: las cárceles femeninas en América Latina. Nueva Sociedad, 208, 73-85.

Azaola, E. (2007). Género y justicia penal en México. En E. Almeda, \& E. Bodelón (Eds.), Mujeres y castigo: un enfoque socio jurídico y de género (pp. 67-82). Madrid: Dykinson.

Ballesteros, A. (2017). Redomesticidad y encarcelamiento femenino en el sistema penitenciario español. Los módulos de respeto. Papers, 102(2), 261-285.doi:http:// dx.doi.org/10.5565/rev/papers.2338.

Birgin, H. (Ed.) (2000a). Las trampas del poder punitivo: el género del derecho penal. Buenos Aires: Biblos.

Birgin, H. (Ed.) (2000b). El derecho en el género y el género en el derecho. Buenos Aires: Biblos.

Bodelón, E. (2003). Género y sistema penal: los derechos de las mujeres en el sistema penal. En R. Bergalli (Ed.), Sistemas 
penales y problemas sociales (pp. 451486). Valencia: Tirant lo Blanch.

Bodelón, E., \& Aedo, M. (2015). Las niñas en el sistema de justicia penal. Anales de la Cátedra Francisco Suárez, 49, 219-236.

Brown, M.; Chesney-Lind, M., \& Stein, N. (2007). Patriarchy matters: Toward a gendered theory of teen violence and victimization. Violence Against Women, 13(12), 328-345.

Bryman, A. (2001). Ethnography. Londres: Sage.

Butler, J. (2001). El género en disputa: el feminismo y la subversión de la identidad. México, D. F.: Paidós.

Butler, J. (2002). Cuerpos que importan. Buenos Aires: Paidós.

Butler, J. (2006). Deshacer el género. Barcelona: Paidós.

Carlen, P., \& Worrall, A. (2004). Analysing women 's imprisonment. Londres: Willan.

Chesney-Lind, M. (1989). Girls' crime and womans' place: Toward a feminist model of female delinquency. Crime \& Delinquency, 35(1), 5-29.

Chesney-Lind, M., \& Pasko, L. (2013). Girls, women, and crime. California: Sage.

Cladem (2016). Niñas madres. Embarazo y maternidad infantil forzada en América Latina y el Caribe. Balance regional. Asunción: Cladem. Recuperado de: www. cladem.org.

Comité de los Derechos del Niño. (2015). Observaciones finales sobre los informes periódicos tercero a quinto combinados del Uruguay. Montevideo: Naciones Unidas. Recuperado de: http://www.comitednu. org/wp-content/uploads/2015/04/ O B S E R VA C I O N E S - F I N A L E S ESPA\%C3\%91OL-CRC.pdf.

Crenshay, K. (2004). Interseccionalidad: una herramienta para la justicia de género $\mathrm{y}$ la justicia económica. Derechos de las Mujeres y Cambio Económico, 9, 1-8.

Daly, K. (2013). Different ways of conceptualizing sex/gender in feminist theory and their implications for criminology. En M. Chesney-Lind, \& L. Pasko (Eds.), Girls, women and crime:
Selected readings (pp. 3-19). Los Ángeles: Sage.

De Laurentis, T. (1989). Technologies of gender: Essays on theory, film and fiction. Londres: Macmillan.

De Miguel, E. (2017). Explorando la agencia de las mujeres encarceladas a través de sus experiencias amorosas. Papers, 102(2), 311-335. doi:http://dx.doi.org/10.5565/ rev/papers.2340.

Denzin, N., \& Lincoln, I. (2003). The landscape of qualitative research. Theories and issues. Thousand Oaks: Sage.

Donzelot, J. (1977/ 2008). La policía de las familias. Buenos Aires: Nueva Visión.

Fernández, A. M. (1993). La invención de la niña. Buenos Aires: Unicef-Xerox.

Ferrajoli, L. (2003). Prólogo. En P. Tamar (Ed.), Un derecho para dos: la construcción jurídica de género, sexo y sexualidad (pp. 11-17). Madrid: Trotta.

Fessler, D. (1998). Niños y menores, la asistencia de los "hijos del pueblo". (Trabajo final monográfico de Licenciatura en Historia). Universidad de la República, Montevideo, Uruguay.

Foucault, M. (1991). La verdad y las formas jurídicas. Barcelona: Gedisa.

Fries, L., \& Matus, V. (2000). La ley hace al delito. Santiago de Chile: LOM, La Morada.

Galeotti-Galmés, R. (2013). Adolescentes infractoras: discursos y prácticas del Sistema Penal Juvenil Uruguayo. Montevideo: Psicolibros-Waslala.

González, C. (2015). La infracción adolescente a la ley penal en el centro del debate sobre seguridad pública en Uruguay. Revista Latinoamericana de Ciencias Sociales, Niñez y Juventud, 13(2), 951-962. doi:10 $.11600 / 1692715 x .13228201014 \quad$ Acceso $(19 / 05 / 2017)$.

González, C., Leopold, S., López-Gallego, L., \& Martinis, P. (2013). Los sentidos del castigo: el debate uruguayo sobre la responsabilidad en la infracción adolescente. Montevideo: Trilce.

Hammersley, M. (2007). Ethnography: principles in practice. Londres: Routledge.

Haraway, D. (1995). Ciencia, cyborgs y 
mujeres: la reinvención de la naturaleza. Madrid: Cátedra.

Harding, S. (1987). Is There a Feminist Method? Recuperado de: http://goodliffe. byu.edu/310/protect/harding1.pdf.

Harding, S. (1996). Ciencia y Feminismo. Madrid: Morata.

Hernández, F. (2013). Cárceles de mujeres del novecientos: una práctica de siglos. En P. Oliver (Coord.), El siglo de los castigos: prisión y formas carcelarias en la España del siglo $X X$. Madrid: Anthropos.

Hochschild,A.(2003/2011).Lamercantilización de la vida intima: apuntes de la casa y el trabajo. Madrid: Katz.

Igareda, N. (2009). La maternidad de las mujeres presas. En N. Gemma, \& E. Bodelon (Eds.), Género y Dominación 7: críticas feministas del derecho y el poder (pp. 159194). Barcelona: Anthropos, OSPDH.

Institución Nacional de Derechos Humanos y Defensoría del Pueblo de la República Oriental del Uruguay. (2015). Informe de seguimiento sobre la situación de las adolescentes privadas de libertad en el Centro de Ingreso de Adolescentes Femenino (Ciaf) al 19 de mayo de 2015. Recuperado de: http://inddhh.gub.uy/wpcontent/uploads/2015/07/043.-InformeCiaf-07-07-2015.pdf.

Juliano, D. (2009). Delito y pecado: la transgresión en femenino. Política $y$ Sociedad, 46(1-2), 79-95.

Katzkowicz, S., La Buonora, L., Pieri, D., Pandolfi, J., Semblat, F., Nuñez, S., Sauval, M., \& Thevenet, N. (2015). El trabajo de cuidados desde una perspectiva de género y generaciones. Montevideo: Inju-Mides.

Lagarde, M. (2014). Los cautiverios de las mujeres: madresposas, monjas, putas, presas y locas. México, D. F.: Siglo Veintiuno, Unam.

Larrandart, L. (2000). Control social, derecho penal y género. En H. Birgin (Ed.), Las trampas del poder punitivo: el género del derecho penal (pp. 85-109). Buenos Aires: Biblos.

Larrauri, E. (Ed.) (1994). Mujeres, derecho penal y criminología. Madrid: Siglo
Veintiuno.

Leopold, S. (2014). Los laberintos de la infancia: discursos, representaciones y crítica. Montevideo: Ediciones Universitarias.

López-Gallego, L. (2011). Géneros de Encierro: Cuando las Adolescentes son las "Internadas". "Ponencia presentada en las X Jornadas de Investigación, Facultad de Ciencias Sociales, Universidad de la República", Montevideo, Uruguay.

López-Gallego, L. (2016). Prácticas de control socio-penal: dispositivo psi pericial y adolescentes mujeres en el sistema penal juvenil uruguayo. (Tesis para obtener el título de Doctora). Universitat Autònoma de Barcelona, Barcelona, España. Recuperado de: http://www.tdx.cat/ handle/10803/368186

López-Gallego, L., \& Montes-Maldonado, C. (2016). Domesticidad y cuidados. Acerca de las adolescentes mujeres en su tránsito por los sistemas penales juveniles. En Casa Bertolt Brecht. (Comp.), Adolescentes y privación de libertad en Uruguay: aportes académicos al debate. Montevideo: Fundación Rosa Luxemburgo.

López-Gómez,A.(Coord.)(2005).Adolescentes $y$ sexualidad. Significados, discursos $y$ acciones en Uruguay. Montevideo: Udelar, UNFPA.

López-Gómez, A., \& Varela, C. (2016). Maternidad en adolescentes y desigualdad social en Uruguay. Análisis territorial desde la perspectiva de sus protagonistas. Montevideo: UNFPA, Udelar.

Miller, J. (2003). Gender, crime and (in)justice. Journal of Contemporary Ethnography, 32(1), 3-8.

Morás, L. E. (1992). Los hijos del Estado: fundación y crisis del modelo de protección-control de menores en Uruguay. Montevideo: Serpaj-Departamento de Sociología de la Facultad de Ciencias Sociales.

Nicolás-Lazo, G. (2009). Debates en epistemología feminista: del empiricismo y el standpoint a las críticas postmodernas sobre el sujeto y el punto de vista. En G. Nicolás-Lazo, \& E. Bodelón (Comps.), Género y dominación: críticas del 
derecho y el poder (pp. 25-62). Barcelona: Anthropos.

Pasko, L. (2013). Setting the record straight: Girls, sexuality, and the juvenile correctional system. En M. Chesney-Lind, \& L. Pasko (Eds.), Girls, women and crime: Selected readings (pp. 215-227). Los Ángeles: Sage.

Pasko, L., \& López, V. (2015). The Latina penalty: Juvenile correctional attitudes toward the Latina juvenile offender. Journal of Ethnicity in Criminal Justice, 13(1), 1-20. doi:10.1080/15377938.2015. 1015196.

Pitch, T. (2003). Un derecho para dos: la construcción jurídica de género, sexo y sexualidad. Madrid: Trotta.

Pitch, T. (2009). La sociedad de la prevención. Buenos Aires: Ad-Hoc.

Portillo, Á. (1989). Estado y minoridad en el Uruguay. Montevideo: Roca Viva.

Reguillo, R. (2000). Emergencia de las culturas juveniles: estrategias del desencanto. Buenos Aires: Norma.

Reyes, R. (1989). Psicología y reeducación de la adolescente. Montevideo: Eppal, CFEE.

Ritchie, J., \& Lewis, J. (2003). Qualitative research practice. A guide for social scince students and researchers. London: Sage.

Rubin, G. (1975). The traffic in women: Notes toward a political economy of sex. En R. Reiter (Ed.), Toward an Antropology of Women (pp. 157-210). Nueva York: Monthly Review.

Scott, J. (1996). El género: una categoría útil para el análisis histórico. En M. Lamas (comp.), El género: la construcción cultural de la diferencia sexual (pp.265302). México, D. F.: Pueg.

Smart, C. (1976). Women, crime and criminology. Londres: Routlegde and Kegan Paul.

Smart, C. (2000). La teoría feminista y el discurso jurídico. En H. Birgin (Ed.), El derecho en el género y el género en el derecho (pp. 31-71). Buenos Aires: Biblos.

Varela, C., \& Lara, C. (2015). Jóvenes de hoy, adolescentes de ayer en Uruguay: maternidad y desempeños. Sociedad y Economía, 29, pp. 15-37. 